Journal of Computer Science 2 (5): 431-433, 2006

ISSN 1549-3636

(C) 2006 Science Publications

\title{
MPLS over Segmented WDM Optical Packet Switching Networks
}

\author{
${ }^{1}$ Hakim Mellah and ${ }^{2}$ Fouad Mohammed Abbou \\ ${ }^{1}$ Faculty of Information Science and Technology (FIST) \\ Multimedia University (MMU), Melaka Campus, Malaysia \\ ${ }^{2}$ Alcatel Network Systems (Malaysia), Multimedia University, Cyberjaya Campus
}

\begin{abstract}
Wavelength Division Multiplexing (WDM) is a promising solution for data transport in future all-optical wide area networks. Such networks consist of fibers joined by dynamically controllable cross-connects which provide purely optical transport between pairs of network access stations. Optical packet switching (OPS) is optical switching with the finest granularity. Incoming packets are switched all-optically without being converted to electrical signal. There are two categories of OPS networks. Slotted (synchronous) OPS networks, in which all the packets have the same size and unslotted (asynchronous) OPS networks, where packets may or may not have the same size. In this study we propose to integrate MPLS over slotted OPS networks by aggregating optical packets into a labeled optical burst. The burst has a fixed number of packets (segments). The number of segments in each burst is encoded in the experimental field of the MPLS header.
\end{abstract}

Keywords: MPLS, OPS, WDM

\section{INTRODUCTION}

The deployment of Wavelength-Division Multiplexing (WDM) in communications networks has brought solutions to satisfy the rapidly increasing demand for the bandwidth capacity introduced by the huge explosion in the public Internet ${ }^{[1]}$. This situation led to research interest in optical packet switching (OPS), which appears to be a strong candidate because of the high speed, data rate/format transparency and configurability it offers ${ }^{[2,3]}$. In general, optical packet switched networks are divided into two categories: slotted (synchronous) and unslotted (asynchronous) ${ }^{[2]}$.

In a slotted OPS network all the packets have the same size. They are placed together with the header inside a fixed time slot, having a longer duration than the header and the packet to provide a guard time before and after each packet ${ }^{[2]}$. The architecture of a typical OPS node in a slotted network is shown in Fig. $1^{[4]}$.

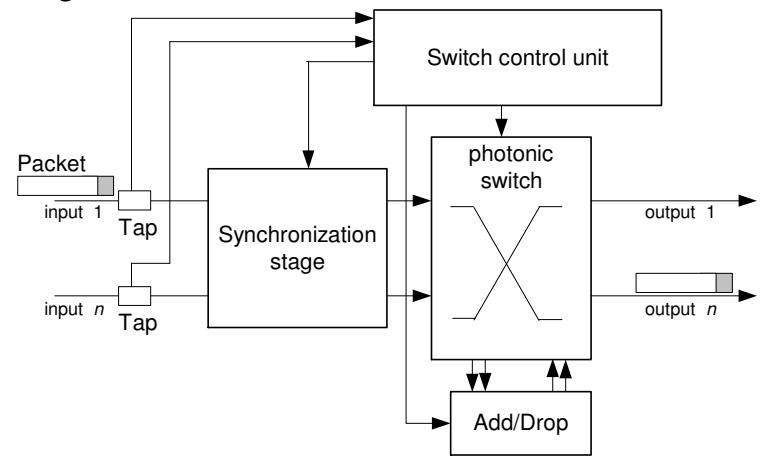

Fig. 1:Generic node architecture of the slotted OPS network
$\operatorname{MPLS}^{[5,6]}$ is a key development in Internet technologies that will assist in adding a number of essential capabilities to today's best effort IP-based networks. It replaces the standard destination-based hop-by-hop forwarding paradigm in IP-based networks with a label swapping-forwarding paradigm. This has the benefits of simplifying the packet-forwarding engine $^{[7]}$, enabling easy scaling to terabit rates and enhancing service provisioning capabilities ${ }^{[8]}$. Furthermore, it decouples forwarding from routing ${ }^{[5-7]}$, enabling one to apply new specialized or customized routing services without requiring changes in the forwarding path.

In an MPLS-based network, once a packet is received by an edge label switching router (LSR), the packet is examined and a label is assigned to it.

At subsequent routers, there is no further analysis of the packet's network layer header ${ }^{[5]}$. Rather, the entire journey of the packet inside the MPLS-based network will be based on the label only.

There has been recent interest in using label swapping applied directly in the optical domain to perform $\mathrm{OPS}^{[9]}$. The proposed advantages are that OPS will provide finer granularity for an Optical Transport Network than wavelength switching while maintaining the performance advantage of photonic switching over electronic switching alternatives ${ }^{[10,11]}$.

\section{OPTICAL MPLS}

Figure 2 illustrates an optical MPLS (OMPLS) network. Packets from different source nodes enter the core network at an ingress node. Packets with the same

Corresponding Author: Hakim Mellah, Faculty of Information Science and Technology (FIST), Multimedia University (MMU), Melaka Campus, Malaysia 
destination address are aggregated into one burst. A burst can consist of up to 8 packets. A label is associated with the burst and a MPLS header is then generated and added to it. An End Of Burst Sequence (EOBS) is also added to the burst indicating the end of the burst in case a length mismatch because of contention resolution. The number of packets in a burst is enough to determine the burst size since all the packets have the same size. The number of packets in the burst is encoded in the Experimental field of the MPLS header shown in Fig. $3^{[12]}$.

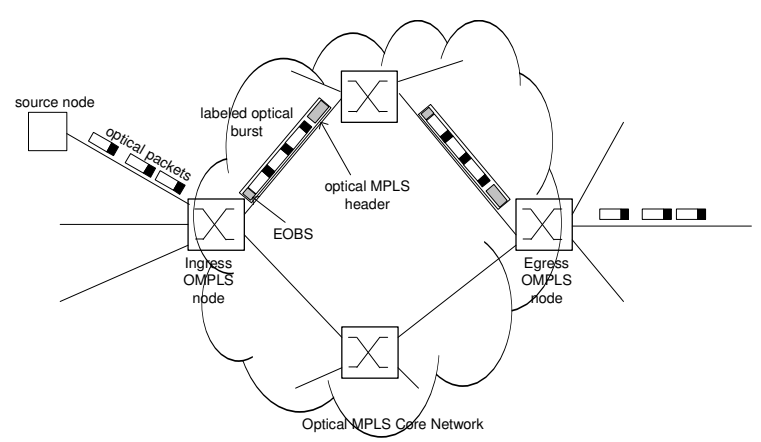

Fig. 2: Optical MPLS core network

\begin{tabular}{|c|c|c|l|}
\hline Label & Exp & BS & TTL \\
\hline 20-bits & 3-bits & 1-bit & 8-bits \\
\hline
\end{tabular}

Fig. 3: MPLS header

Once inside the core network, core routers computes a new label and wavelength from a routing table given the current label, current wavelength and fiber port ${ }^{[13]}$. The original label is then swapped with the new label and the labeled burst is converted to the new wavelength.

At the egress node, the optical MPLS header is removed from the burst and then the individual packets are extracted and sent to their destination.

The optical label coding can be realized with the serial label coding ${ }^{[13]}$ illustrated in Fig. 4. where a fixed bit rate MPLS label is multiplexed at the head of the optical burst with the two separated by an optical guard-band $^{[13]}$.

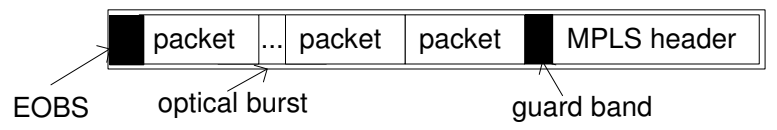

Fig. 4: Optical label coding

\section{THE PROPOSED BURST SIZE REPRESENTATION}

MPLS header experimental field: The MPLS header shown in Fig. 3 contains a 20-bit label, a 3-bit experimental field, a 1-bit stack indicator and an 8-bit time-to-live (TTL) field. The header doesn't provide the packet size which means either another header should be examined, or all the packets have the same size. However, the experimental field can be used as an indication of the labeled packet (burst) size $L$. In the slotted OPS network, all the packets have the same size; hence the size of a burst consisting of $n$ packets is equal to $n L$. In the proposed scheme, a burst can consist of up to 8 packets, which can be encoded using the 3-bit exp field in the MPLS header, with 000 indicating an 8 packets burst, 001 a 1 packet burst and 111 a 7 packets burst.

Contention resolution and EOBS: When the core switch receives a burst, it extracts its MPLS header, computes a new label and wavelength from a routing table given the current label, current wavelength and fiber port ${ }^{[13]}$. The original label is then swapped with the new label and the labeled burst is converted to the new wavelength. The burst size is computed from the exp field and the packet size. A copy of the new MPLS header is saved and the burst starts its way out. In case of a low priority burst is interrupted by a high priority burst while in transmission, the switch will continue transmitting the current packet of the low priority burst, inserting an EOBS and then saving the remaining packets. After that, the high priority burst will be switched out. The switch then updates the MPLS header of the low priority burst, add it to the rest of the packets forming a new burst and switched out once the output port is available as shown in Fig. 5.
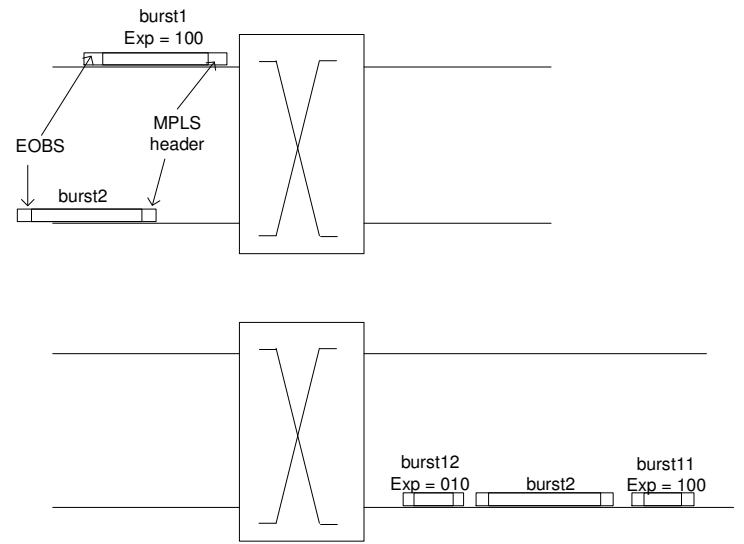

Fig. 5: Contention resolution in labeled optical burst

\section{PERFORMANCE EVALUATION OF LABELED SEGMENTED WDM OPS}

Burst blocking probability: Aggregating packets doesn't modify the blocking probability of the system. With the assumption that the packet arriving process at a given output port of the switch is a Poisson process with rate $\lambda$, packet transmission time $1 / \mu$, the number of wavelengths on the output fiber is $K$ and there is no extra waiting buffers, the switch is a bufferless system which can be modeled as an $M / M / K / K$ queue ${ }^{[14]}$. The packet blocking probability is given by the following Erlang B formula: 


$$
P B_{1}=\frac{\frac{(\lambda / \mu)^{K}}{K !}}{\sum_{m=0}^{K} \frac{(\lambda / \mu)^{m}}{m !}}
$$

Now, if $N$ packets are aggregated to form a burst, then the system still can be modeled as an $M / M / K / K$ queue with a Poisson arrival with a rate of $\lambda / N$ and the average transmission time required for each burst is $N / \mu$. The new burst blocking probability $\mathrm{PB}_{2}$ is equal to the packet blocking probability $\mathrm{PB}_{1}$ given in equ. 1 above.

Synchronization and header processing overhead reduction: The overhead cost due to synchronizing, processing, routing, differentiation of services is the same irrespective of the size of the packet ${ }^{[15]}$. Hence, reducing the number of packets in the network therefore improves overall performance.

\section{CONCLUSION}

In this study we have proposed to use MPLS in synchronous optical packet switching networks where packets to be delivered to the same destination are aggregated in a single burst and labeled. The switching of the burst in subsequent nodes will depend on the current label. The burst size is encoded in the MPLS exp field. This field is used as an indicator to the number of packets in the burst, which can be up to 8 since the field has only 3 bits. The packets in a synchronous OPS network have the same size, so the burst size is determined by knowing the number of packets composing the burst. The proposed scheme can also be used for contention resolution scenarios, since the burst consist of an integer number of packet which are well delimited. Aggregating packets doesn't modify the blocking probability of the system and reducing the number of packets in the network therefore improves overall performance.

\section{REFERENCES}

1. Choi, Y.B., J.Y. Kim and K.Y. Kim, 2000. Wavelength-division multiplexed optical packet networks for TCP-IP backbone. IEEE TENCON, pp: 493-497.
2. Yao, S., B. Mukherjee and S. Dixit, 2000. Advances in photonic packet switching: an overview. IEEE Commun. Mag., 38: 84-94.

3. El-Bawab, T.S. and J.D. Shin, 2002. Optical packet switching in core networks: between vision and reality. IEEE Commun. Mag., 40: 60-65.

4. Yao, S., B. Mukherjee, S.J.B. Yoo and S. Dixit, 2003. A unified study of contention-resolution schemes in optical packet-switched networks. IEEE J. Lightwave Technol., 21: 672-683.

5. LeFaucheur, F., 1998. IETF Multiprotocol Label Switching (MPLS) Architecture. IEEE, pp: 6-15.

6. Lawrence, J., 2001. Designing Multiprotocol Label Switching Networks. IEEE Commun. Mag., pp: 134-142.

7. Viswanathan, A. et al., 1998. Evolution of multiprotocol label switching. IEEE Commun. Mag., pp: 165-173.

8. Ayyangar, A. and D. Sidhu, 2001. Analysis of MPLS based traffic engineering solution. Joint 4th IEEE Intl. Conf. on ATM (ICATM 2001) and High Speed Intelligent Internet Symp., pp: 21-27.

9. Blumenthal, D.J., B.E. Olsson, G. Rossi, T.E. Dimmick, L. Ran, M. Masanovi, O. Lavrova, R. Doshi, O. Jerphagnon, J.E. Bowers, V. Kaman, L.A. Coldren and J. Barton, 2000. All-optical label swapping networks and technologies. J. Lightwave Technol., 18: 2058-2075.

10. O'Mahony, M., D. Simeonidou, D. Hunter and A. Tzanakaki, 2001. The application of optical packet switching in future communication networks. IEEE Commun. Mag., 39: 128-135.

11. Reed, M.J., 2003. MPLS label space for optical packet switched networks. IEEE Intl. Conf. on Commun., 2: 1273-1277.

12. Xiao, X. et al., 2000. Traffic engineering with MPLS in the internet. IEEE Network, pp. 28-33.

13. Blumenthal, D.J. et al., 2000. All-optical label swapping networks and technologies. IEEE J. Lightwave Technol., 18: 2058-2075.

14. Roseberg, Z., H.L. Vu and M. Zukerman, 2003. Burst segmentation benefit in optical switching. IEEE Comm. Lett., 7: 127-129.

15. Tounsi, H., L. Toutain and F. Kamoun, 2001. Small packets aggregation in an IP domain. Proc. of the 6th IEEE Symp. on Computers and Commun. (ISCC'01), pp: 708-713. 